\title{
On a transmission problem in elasticity
}

\author{
by Christodoulos Athanasiadis and \\ IOANNIS G. STRATis (Athens)
}

\begin{abstract}
The transmission problem for the reduced Navier equation of classical elasticity, for an infinitely stratified scatterer, is studied. The existence and uniqueness of solutions is proved. Moreover, an integral representation of the solution is constructed, for both the near and the far field.
\end{abstract}

1. Introduction. In this work we are studying the transmission problem for the reduced Navier equation, in the case where a plane elastic wave is incident upon a nested body of an infinite number of homogeneous layers. On the surfaces that describe this tessellation, the transmission conditions are imposed that express the continuity of the medium, and the equilibrium of the forces acting on it.

In [15] Sabatier reviews (in the framework of the so-called impedance equation) available answers to the question whether modelling media by continuous, or piecewise constant, parameters leads to essential modifications in the behaviour of scattering problems; see also the references therein. The general theory of scattering of elastic waves is very well presented by Kupradze [12], [13], who discusses many interesting quantitative, as well as qualitative, aspects of elastic wave propagation and scattering. The present work is strongly affected by the above references. For the description of the mathematical theory of classical elasticity we also refer to [7].

Uniqueness theorems are, among others, proved by Jones [10] and Wheeler and Sternberg [16], who also present integral representations for the displacement field. Existence theorems are presented in [12], [13] by potential methods, and in [8] by variational methods. Low-frequency elastic scattering has been studied by Dassios and Kiriaki [6] for the case of a single scatterer, by Kiriaki and Polyzos [11] for a penetrable body with an impene-

1991 Mathematics Subject Classification: 73C02, 73C15, 35Q72, 35R05.

Key words and phrases: transmission problem, infinitely stratified scatterer, scattering amplitudes. 
trable core, and by Jones [9] for a single scatterer, where an explicit formula for the second level of approximation is obtained. The general framework, and results about wave propagation in dissipative materials, may be found in the recent book by Caviglia and Morro [5], where a rich bibliography is also included; in particular, transmission and scattering problems are studied there, and many applications of such problems are encountered.

In Section 2, first we formulate the transmission problem. Then we prove that the corresponding homogeneous transmission problem has as its only classical solution the trivial one; this is done by a method based on the basic energy theorem, [12]. Next, by a generalized solutions approach, the existence and uniqueness of a weak solution to the non-homogeneous transmission problem is proved. This solution is shown to be classical by a regularity argument. Such an approach has been used by the authors in [1] for transmission problems in acoustics, in [2] for parabolic and hyperbolic diffraction problems, and in [3] for elliptic transmission problems.

In Section 3, we construct an integral representation of the solution, in which the transmission conditions and the radiation conditions are incorporated, and we study the asymptotic behaviour of the scattered wave in the radiation region.

Finally, in Section 4, a number of comments have been included on the relation of our results to previous research. The heavily technical parts of proofs of results in Sections 2 and 3 are included in the Appendix.

2. The transmission problem. Let $\Omega$ be a bounded, convex subset of $\mathbb{R}^{3}$, with $\mathbf{0} \in \Omega$, and $S_{0}=\partial \Omega$ is supposed to be a 2-dimensional $C^{2}$-surface. The exterior, $\Omega_{0}$, of $\Omega$ is an infinite homogeneous isotropic elastic medium with Lamé constants $\lambda_{0}, \mu_{0} . \Omega$ is considered to be a bonded nested piecewise homogeneous body, consisting of annuli-like regions $\Omega_{j}$, divided by 2-dimensional $C^{2}$-surfaces $S_{j}, j=1,2, \ldots$, where $S_{j}$ surrounds $S_{j+1}$. We assume that $\operatorname{dist}\left(S_{j-1}, S_{j}\right)>0$ for all $j=1,2, \ldots$ Let $\lambda_{j}, \mu_{j}$ be the Lamé constants in the layer $\Omega_{j}, j=1,2, \ldots$ By the adjective "bonded" it is meant that the displacement and traction are continuous across each $S_{j}$, as will be guaranteed by the transmission conditions. Moreover, we assume that $\sum_{j=1}^{\infty}\left|S_{j}\right|<\infty$, where $\left|S_{j}\right|$ is the measure of $S_{j}$. Such a scatterer will be referred to as an infinitely stratified scatterer. This stratified structure allows a variety of applications in biology and geophysics.

We consider the standard operators of linear elasticity

$$
\begin{aligned}
\Delta_{j}^{*} & :=\mu_{j} \Delta+\left(\lambda_{j}+\mu_{j}\right) \operatorname{grad} \operatorname{div}, \\
T_{j} & :=2 \mu_{j} \frac{\partial}{\partial n}+\lambda_{j} \widehat{\mathbf{n}} \operatorname{div}+\mu_{j} \widehat{\mathbf{n}} \times \operatorname{curl},
\end{aligned}
$$

for all $j=0,1,2, \ldots$, where $\Delta$ denotes the Laplacian. 
Suppose that a time-harmonic plane wave $\psi(\mathbf{r})$ of angular frequency $\omega$ is incident upon a scatterer of the above form, resulting in the emanation of a scattered wave $\mathbf{u}_{0}(\mathbf{r})$. The total exterior field, $\psi_{0}(\mathbf{r})$, in $\Omega_{0}$, is the superposition of the incident and scattered fields:

$$
\boldsymbol{\psi}_{0}(\mathbf{r})=\boldsymbol{\psi}(\mathbf{r})+\mathbf{u}_{0}(\mathbf{r}) .
$$

The longitudinal part $\mathbf{u}_{0}^{\mathrm{p}}$ and the transverse part $\mathbf{u}_{0}^{\mathrm{s}}$ of the scattered field satisfy the radiation conditions

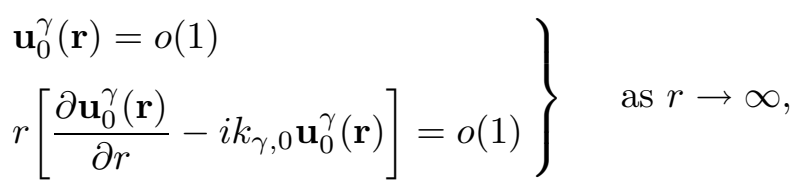

uniformly for all directions $\widehat{\mathbf{r}}$, where $k_{\gamma, 0}$ denotes the wave number of the incident $\gamma$-wave, $\gamma=\mathrm{p}$, s, respectively.

The mathematical description of the above situation leads to a transmission problem of the following form: Find $\mathbf{u}$ satisfying

$$
\begin{aligned}
& \Delta_{0}^{*}\left(\boldsymbol{\Psi}+\mathbf{u}_{0}\right)+\omega^{2}\left(\boldsymbol{\psi}+\mathbf{u}_{0}\right)=\mathbf{0} \quad \text { in } \Omega_{0}, \\
& \left.\begin{array}{l}
\Delta_{j}^{*} \mathbf{u}_{j}+\omega^{2} \mathbf{u}_{j}=\mathbf{0} \quad \text { in } \Omega_{j}, j=1,2, \ldots, \\
\left.\begin{array}{l}
\boldsymbol{\psi}+\mathbf{u}_{0}=\mathbf{u}_{1} \\
T_{0}\left(\boldsymbol{\psi}+\mathbf{u}_{0}\right)=T_{1} \mathbf{u}_{1}
\end{array}\right\} \quad \text { on } S_{0}, \\
\mathbf{u}_{j}=\mathbf{u}_{j+1} \\
T_{j} \mathbf{u}_{j}=T_{j+1} \mathbf{u}_{j+1}
\end{array}\right\} \quad \text { on } S_{j}, j=1,2, \ldots,
\end{aligned}
$$

together with the radiation conditions $(2.4),(2.5)$, where $\mathbf{u}_{j}$ denotes the restriction of $\mathbf{u}$ to $\Omega_{j}, j=0,1,2, \ldots$

It is well known that the incident field $\boldsymbol{\psi}$ satisfies $\Delta_{0}^{*} \boldsymbol{\psi}+\omega^{2} \boldsymbol{\psi}=\mathbf{0}$ in $\Omega_{0}$. Therefore, the above transmission problem can be written as

$$
\begin{aligned}
& \Delta_{j}^{*} \mathbf{u}_{j}+\omega^{2} \mathbf{u}_{j}=\mathbf{0} \quad \text { in } \Omega_{j}, j=0,1,2, \ldots, \\
& \left.\begin{array}{l}
\mathbf{u}_{1}-\mathbf{u}_{0}=\boldsymbol{\psi} \\
T_{1} \mathbf{u}_{1}-T_{0} \mathbf{u}_{0}=T_{0} \boldsymbol{\psi}
\end{array}\right\} \quad \text { on } S_{0}, \\
& \left.\begin{array}{l}
\mathbf{u}_{j+1}=\mathbf{u}_{j} \\
T_{j+1} \mathbf{u}_{j+1}=T_{j} \mathbf{u}_{j}
\end{array}\right\} \quad \text { on } S_{j}, j=1,2, \ldots,
\end{aligned}
$$

together with (2.4) and (2.5).

This transmission problem will be denoted by (NHTP) in the sequel, while the corresponding homogeneous transmission problem, i.e. when (2.12) holds for all $j=0,1,2, \ldots$, will be denoted by (HTP).

We are now in a position to prove

TheOrem 2.1. (HTP) has only the trivial solution. 
Proof. Let $\Omega_{0, R}=\{\mathbf{r}: r<R\}, R>0$, be a ball in $\mathbb{R}^{3}$, circumscribed around $\Omega$. By the energy theorem, [12], applied in $\Omega_{0, R}-\Omega$, we have

$$
\frac{\partial}{\partial t}\left(U_{0}+K_{0}\right)=\int_{r=R}\left(\frac{\partial \mathbf{v}}{\partial t} \cdot T_{0} \mathbf{v}\right) d s-\int_{S_{0}}\left(\frac{\partial \mathbf{v}}{\partial t} \cdot T_{0} \mathbf{v}\right) d s
$$

where $\mathbf{v}(\mathbf{r}, t)=\operatorname{Re}[\mathbf{u}(\mathbf{r}) \exp (-i \omega t)], \mathbf{u}(\mathbf{r})$ being a solution of (HTP).

Similarly, in $\Omega_{1}$, we have

$$
\frac{\partial}{\partial t}\left(U_{1}+K_{1}\right)=\int_{S_{0}}\left(\frac{\partial \mathbf{v}}{\partial t} \cdot T_{1} \mathbf{v}\right) d s-\int_{S_{1}}\left(\frac{\partial \mathbf{v}}{\partial t} \cdot T_{1} \mathbf{v}\right) d s .
$$

By the transmission conditions, we have

$$
T_{0} \mathbf{v}=T_{1} \mathbf{v} \quad \text { on } S_{0},
$$

and, hence, we arrive at

$$
\begin{aligned}
\frac{\partial}{\partial t}\left(U_{0}+K_{0}\right)+\frac{\partial}{\partial t}\left(U_{1}\right. & \left.+K_{1}\right) \\
& =\int_{r=R}\left(\frac{\partial \mathbf{v}}{\partial t} \cdot T_{0} \mathbf{v}\right) d s-\int_{S_{1}}\left(\frac{\partial \mathbf{v}}{\partial t} \cdot T_{1} \mathbf{v}\right) d s,
\end{aligned}
$$

where $U_{0}, K_{0}$ denote the potential and kinetic energy, respectively, in $\Omega_{0, R}$, and $U_{1}, K_{1}$ in $\Omega_{1}$.

By repeated application of the energy theorem in $\Omega_{j}, j=2,3, \ldots$, we finally get

$$
\frac{\partial}{\partial t}\left(U_{0}+K_{0}\right)+\sum_{j=1}^{\infty} \frac{\partial}{\partial t}\left(U_{j}+K_{j}\right)=\int_{r=R}\left(\frac{\partial \mathbf{v}}{\partial t} \cdot T_{0} \mathbf{v}\right) d s
$$

whence, using Lemma A.1 (see Appendix), we have

$$
\frac{\partial}{\partial t}\left[\left(U_{0}+K_{0}\right)+\sum_{j=1}^{\infty}\left(U_{j}+K_{j}\right)\right]=\int_{r=R}\left(\frac{\partial \mathbf{v}}{\partial t} \cdot T_{0} \mathbf{v}\right) d s .
$$

By Lemma A.2 (Appendix), from (2.18) it follows that $\mathbf{u}_{0}(\mathbf{r})=\mathbf{0}$ in $\Omega_{0}$. We now proceed to show that $\mathbf{u}_{1}(\mathbf{r})=\mathbf{0}$ in $\Omega_{1}$. This having been accomplished, $\mathbf{u}_{2}(\mathbf{r})$ will be equal to zero in $\Omega_{2}$, too, etc. By (A35) (Appendix) and the transmission conditions on $S_{0}$, we are led to the problems

$$
\left\{\begin{array}{l}
\Delta \mathbf{u}_{1}^{\mathrm{p}}+k_{\mathrm{p}, 1}^{2} \mathbf{u}_{1}^{\mathrm{p}}=\mathbf{0} \quad \text { in } \Omega_{1}, \\
\mathbf{u}_{1}^{\mathrm{p}}=\mathbf{0} \\
T_{1} \mathbf{u}_{1}^{\mathrm{p}}=2 \mu_{1} \frac{\partial \mathbf{u}_{1}^{\mathrm{p}}}{\partial n}+\mathbf{n} \lambda_{1} \operatorname{div} \mathbf{u}_{1}^{\mathrm{p}}=\mathbf{0}
\end{array}\right\} \quad \text { on } S_{0}
$$


and

$$
\left\{\begin{array}{l}
\Delta \mathbf{u}_{1}^{\mathrm{s}}+k_{\mathrm{s}, 1}^{2} \mathbf{u}_{1}^{\mathrm{s}}=\mathbf{0} \quad \text { in } \Omega_{1}, \\
\mathbf{u}_{1}^{\mathrm{s}}=\mathbf{0} \\
T_{1} \mathbf{u}_{1}^{\mathrm{s}}=2 \mu_{1} \frac{\partial \mathbf{u}_{1}^{\mathrm{s}}}{\partial n}+\mu_{1}\left(\mathbf{n} \times \operatorname{curl} \mathbf{u}_{1}^{\mathrm{s}}\right)=\mathbf{0}
\end{array}\right\} \quad \text { on } S_{0} .
$$

Direct computation shows that

$$
T_{1} \mathbf{u}_{1}^{\mathrm{p}}=\sum_{q=1}^{3} C_{q} \frac{\partial \mathbf{u}_{1}^{\mathrm{p}}}{\partial x_{q}}
$$

and

$$
T_{1} \mathbf{u}_{1}^{\mathrm{s}}=\sum_{q=1}^{3} D_{q} \frac{\partial \mathbf{u}_{1}^{\mathrm{s}}}{\partial x_{q}}
$$

where $C_{q}=\left(c_{i m}^{(q)}\right), i, m=1,2,3$, is given by $c_{i i}^{(q)}=n_{q}\left(2 \mu_{1}+\lambda_{1} \delta_{i q}\right)$ and $c_{i m}^{(q)}=n_{i} \lambda_{1} \delta_{m q}, \delta_{i q}$ being the Kronecker symbol, and $\mathbf{n}=\left(n_{1}, n_{2}, n_{3}\right)$, and $D_{q}=\left(d_{i m}^{(q)}\right), \quad i, m=1,2,3$, is given by $d_{i i}^{(q)}=n_{q}\left(1+\delta_{i q}\right) \mu_{1}$ and $d_{i m}^{(q)}=$ $n_{m} \mu_{1} \delta_{i q}$.

In view of $(2.21),(2.22)$, the problems $(2.19),(2.20)$ are set in the standard form of Cauchy problems, for systems of second order elliptic equations. By the form of $C_{q}, D_{q}, q=1,2,3$, it is apparent that (2.21), (2.22) do not represent tangential derivatives to $S_{0}$. Therefore we may use Holmgren's uniqueness theorem: since the initial data of (2.19), (2.20) are equal to zero, $\mathbf{u}_{1}^{\mathrm{p}}\left(\right.$ resp. $\mathbf{u}_{1}^{\mathrm{s}}$ ) must be equal to zero in $\Omega_{1} \cap V_{\mathrm{p}}$ (resp. $\Omega_{1} \cap V_{\mathrm{s}}$ ), where $V_{\mathrm{p}}$ (resp. $V_{\mathrm{s}}$ ) is a neighbourhood of any point of $S_{0}$. Since $\mathbf{u}_{1}^{\mathrm{p}}$ (resp. $\mathbf{u}_{1}^{\mathrm{s}}$ ) is analytic in $\Omega_{1}$, by the unique continuation principle, it follows that $\mathbf{u}_{1}^{\mathrm{p}} \equiv \mathbf{0}$ (resp. $\left.\mathbf{u}_{1}^{\mathrm{s}} \equiv \mathbf{0}\right)$ in $\Omega_{1}$. Hence $\mathbf{u} \equiv \mathbf{0}$ in $\Omega_{1}$, and the proof is complete.

We now proceed to the solvability of (NHTP).

It is convenient to reformulate (NHTP) into a transmission problem consisting of non-homogeneous equations and homogeneous transmission conditions, of the form

$$
\left\{\begin{array}{l}
\Delta_{j}^{*} \mathbf{w}_{j}+\omega^{2} \mathbf{w}_{j}=\mathbf{f}_{j} \quad \text { in } \Omega_{j}, j=0,1,2, \ldots, \\
\mathbf{w}_{j+1}=\mathbf{w}_{j} \\
T_{j+1} \mathbf{w}_{j+1}=T_{j} \mathbf{w}_{j} \\
\mathbf{w}_{0} \text { satisfies }(2.4) \text { and }(2.5) .
\end{array}\right.
$$

The problem $(2.23)$ will be denoted by $(\overline{\mathrm{NHTP}})$ in the sequel.

The transformation of (NHTP) into $(\overline{\mathrm{NHTP}})$ is performed as follows: Let

$$
\mathbf{w}_{0}=\mathbf{u}_{0}+\xi_{0} \quad \text { in } \Omega_{0}
$$


where $\xi_{0}$ is the unique solution of the problem

$$
\left\{\begin{array}{l}
\Delta_{0}^{*} \xi_{0}+\omega^{2} \xi_{0}=\mathbf{0} \text { in } \Omega_{0}, \\
T_{0} \xi_{0}=T_{0} \boldsymbol{\psi} \text { on } S_{0} \\
\xi_{0} \text { satisfies }(2.4) \text { and }(2.5)
\end{array}\right.
$$

Let, moreover,

$$
\mathbf{w}_{j}=\mathbf{u}_{j}-\xi_{j} \quad \text { in } \Omega_{j}, j=1,2, \ldots,
$$

where

$$
\xi_{j}(\mathbf{r})=\xi(\mathbf{r}), \quad \mathbf{r} \in \Omega_{j}, j=1,2, \ldots,
$$

and $\xi$ is the extension of $\psi-\xi_{0}$ from $S_{0}$ to $\Omega$, defined by

$$
\xi(\mathbf{r})=\boldsymbol{\psi}(\mathbf{r})-\xi_{0}(\mathbf{r}), \quad T_{1} \xi(\mathbf{r})=\mathbf{0}, \quad \mathbf{r} \in S_{0},
$$

and

$$
\xi_{j+1}(\mathbf{r})=\xi_{j}(\mathbf{r}), \quad T_{j+1} \xi_{j+1}(\mathbf{r})=T_{j} \xi_{j}(\mathbf{r}), \quad \mathbf{r} \in S_{j}, j=1,2, \ldots
$$

Note that since $\boldsymbol{\psi}-\xi_{0} \in\left(C^{2}\left(S_{0}\right)\right)^{3}$, it is evident that $\xi \in\left(C^{2}\left(\bar{\Omega} \backslash \bigcup_{j=1}^{\infty} S_{j}\right) \cap\right.$ $C(\bar{\Omega}))^{3}$. Therefore, the function $\mathbf{f}$ defined by

$$
\mathbf{f}(\mathbf{r})= \begin{cases}\mathbf{0}, & \mathbf{r} \in \Omega_{0}, \\ -\left(\Delta_{j}^{*} \xi_{j}(\mathbf{r})+\omega^{2} \xi_{j}(\mathbf{r})\right), & \mathbf{r} \in \Omega_{j}, j=1,2, \ldots,\end{cases}
$$

is continuous in $\mathbb{R}^{3}$. define

Let $\mu(\mathbf{r})=\mu_{j}, \lambda(\mathbf{r})=\lambda_{j}, \mathbf{w}(\mathbf{r})=\mathbf{w}_{j}(\mathbf{r}), \mathbf{r} \in \Omega_{j}, j=0,1,2, \ldots$, and

$$
\begin{aligned}
E(\mathbf{v}, \mathbf{u})= & \lambda(\mathbf{r}) \operatorname{div} \mathbf{v}(\mathbf{r}) \operatorname{div} \mathbf{u}(\mathbf{r}) \\
& +\mu(\mathbf{r}) \sum_{m, q=1}^{3} \frac{\partial v_{m}(\mathbf{r})}{\partial x_{q}}\left(\frac{\partial u_{m}(\mathbf{r})}{\partial x_{q}}+\frac{\partial u_{q}(\mathbf{r})}{\partial x_{m}}\right)
\end{aligned}
$$

and

$$
\begin{aligned}
& R\left(\Omega_{0}\right):=\left\{\mathbf{u}_{0} \in\left(H_{\mathrm{loc}}^{1}\left(\Omega_{0}\right)\right)^{3}: \mathbf{u}_{0}=\mathbf{u}_{0}^{\mathrm{p}}+\mathbf{u}_{0}^{\mathrm{s}}, \mathbf{u}_{0}^{\gamma}=o(1)\right. \text { and } \\
&\left.\partial \mathbf{u}_{0}^{\gamma} / \partial r-i k_{\gamma, 0} \mathbf{u}_{0}^{\gamma}=o(1 / r) \text { as } r \rightarrow \infty, \text { for } \gamma=\mathrm{p}, \mathrm{s}\right\} .
\end{aligned}
$$

Definition 2.1. A function $\mathbf{w} \in\left(H^{1}(\Omega)\right)^{3} \cap R\left(\Omega_{0}\right)$ is called a generalized solution of a problem of the form $(\overline{\mathrm{NHTP}})$, for $\mathbf{f} \in\left(L^{2}(\Omega)\right)^{3}$, iff

$$
\int_{\mathbb{R}^{3}} E(\boldsymbol{\varphi}, \mathbf{w}) d x-\omega^{2} \int_{\mathbb{R}^{3}} \mathbf{w}(\mathbf{r}) \cdot \boldsymbol{\varphi}(\mathbf{r}) d x=-\int_{\Omega} \mathbf{f}(\mathbf{r}) \cdot \boldsymbol{\varphi}(\mathbf{r}) d x
$$

for every $\boldsymbol{\varphi} \in\left(H^{1}\left(\mathbb{R}^{3}\right)\right)^{3}$ such that $\boldsymbol{\varphi}(\mathbf{r})=O\left(1 / r^{2}\right)$ as $r \rightarrow \infty$.

By standard regularity arguments (cf. [1], [3], [8]), the following result can be proved. 
THEOREM 2.2. Let $\mathbf{w}$ be a generalized solution of $(\overline{\mathrm{NHTP}})$. If $\mathbf{f} \in$ $(C(\bar{\Omega}))^{3}$, then $\mathbf{w} \in\left(C^{2}\left(\mathbb{R}^{3} \backslash \bigcup_{j=1}^{\infty} S_{j}\right) \cap C\left(\mathbb{R}^{3}\right)\right)^{3}$, i.e. w is a classical solution of $(\overline{\mathrm{NHTP}})$.

We are now in a position to prove

TheOrem 2.3. (NHTP) has a unique classical solution.

P r o of. It suffices to prove that $(\overline{\mathrm{NHTP}})$ has a unique classical solution. As in the standard theory, $(\overline{\mathrm{NHTP}})$ may be written in the form

$$
\mathbf{w}+A \mathbf{w}=\mathbf{F},
$$

where $A:\left(H^{1}(\Omega)\right)^{3} \cap R\left(\Omega_{0}\right) \rightarrow\left(H^{1}(\Omega)\right)^{3} \cap R\left(\Omega_{0}\right)$ is a compact operator [8], and $\mathbf{F}$ is the standard extension - given by the Riesz Representation Theorem - of $\mathbf{f}$ in $\left(H^{1}\left(\mathbb{R}^{3}\right)\right)^{3}$. The corresponding homogeneous equation is

$$
\mathbf{w}+A \mathbf{w}=\mathbf{0}
$$

and the corresponding adjoint homogeneous equation is

$$
\mathbf{w}^{*}+A^{*} \mathbf{w}^{*}=\mathbf{0} .
$$

Employing a line of argument analogous to that of [7], we may see that the Fredholm Alternative may be implemented for (2.34)-(2.36). By Theorem 2.1, (2.35) - and hence (2.36) too - has only the trivial solution. Therefore, (2.34) has a unique generalized solution, which-since $\mathbf{f} \in(C(\bar{\Omega}))^{3}$ by $(2.30)$ - is a classical solution, by Theorem 2.2 , thus completing the proof.

3. Integral representations of the exterior field and the scattering amplitudes. In order to construct an integral representation for the total exterior field, near or far, of the scatterer, we make use of the fundamental dyadic solution of equation $\Delta^{*} \mathbf{u}+\omega^{2} \mathbf{u}=\mathbf{0}$ in $\Omega_{0}$, given in [6] by

$$
\begin{aligned}
& \text { (3.1) } \widetilde{G}_{0}\left(\mathbf{r}, \mathbf{r}^{\prime}\right) \\
& =\frac{\exp \left(i k_{\mathrm{p}, 0} R\right)}{\left(\lambda_{0}+2 \mu_{0}\right) k_{\mathrm{p}, 0} R}\left[\left(k_{\mathrm{p}, 0}+\frac{3 i}{R}-\frac{3}{k_{\mathrm{p}, 0} R^{2}}\right) \widehat{\mathbf{R}} \otimes \widehat{\mathbf{R}}-\left(\frac{i}{R}-\frac{1}{k_{\mathrm{p}, 0} R^{2}}\right) \widetilde{I}\right] \\
& \quad-\frac{\exp \left(i k_{\mathrm{s}, 0} R\right)}{\mu_{0} k_{\mathrm{s}, 0} R}\left[\left(k_{\mathrm{s}, 0}+\frac{3 i}{R}-\frac{3}{k_{\mathrm{s}, 0} R^{2}}\right) \widehat{\mathbf{R}} \otimes \widehat{\mathbf{R}}-\left(k_{\mathrm{s}, 0}+\frac{i}{R}-\frac{1}{k_{\mathrm{s}, 0} R^{2}}\right) \widetilde{I}\right],
\end{aligned}
$$

where $\widetilde{I}=\widehat{\mathbf{e}}_{1} \otimes \widehat{\mathbf{e}}_{1}+\widehat{\mathbf{e}}_{2} \otimes \widehat{\mathbf{e}}_{2}+\widehat{\mathbf{e}}_{3} \otimes \widehat{\mathbf{e}}_{3}$ is the identity dyadic, and $\mathbf{R}=\mathbf{r}-\mathbf{r}^{\prime}$.

As always, for the observation vector $\mathbf{r}$, we suppose that its measure, $r$, is greater than the radius of the smallest sphere circumscribable around the scatterer. Since $\mathbf{r}^{\prime}$ is inside the scatterer $\Omega$, there exists $\theta>0$ such that 
$R \geq \theta^{-1}$. In what follows, by $\operatorname{grad} \widetilde{G}_{0}\left(\mathbf{r}, \mathbf{r}^{\prime}\right)$ we mean $\operatorname{grad}_{\mathbf{r}^{\prime}} \widetilde{G}_{0}\left(\mathbf{r}, \mathbf{r}^{\prime}\right)$. First we state the following lemma whose proof may be found in the Appendix.

LEMMA 3.1. The series

$$
\begin{aligned}
& \sum_{j=1}^{\infty} \int_{S_{j-1}} \mathbf{u}_{j}\left(\mathbf{r}^{\prime}\right) \cdot\left(T_{j-1}-T_{j}\right) \widetilde{G}_{0}\left(\mathbf{r}, \mathbf{r}^{\prime}\right) d s\left(\mathbf{r}^{\prime}\right), \\
& \sum_{j=1}^{\infty}\left(1-\frac{\mu_{j}}{\mu_{0}}\right) \int_{\Omega_{j}} \mathbf{u}_{j}\left(\mathbf{r}^{\prime}\right) \cdot \widetilde{G}_{0}\left(\mathbf{r}, \mathbf{r}^{\prime}\right) d v\left(\mathbf{r}^{\prime}\right), \\
& \sum_{j=1}^{\infty}\left(\lambda_{j}-\frac{\lambda_{0}}{\mu_{0}} \mu_{j}\right) \int_{\Omega_{j}} \mathbf{u}_{j}\left(\mathbf{r}^{\prime}\right) \cdot \operatorname{grad} \operatorname{div} \widetilde{G}_{0}\left(\mathbf{r}, \mathbf{r}^{\prime}\right) d v\left(\mathbf{r}^{\prime}\right)
\end{aligned}
$$

converge uniformly.

We denote by $\boldsymbol{\sigma}_{1}(\mathbf{r}), \boldsymbol{\sigma}_{2}(\mathbf{r})$ and $\boldsymbol{\sigma}_{3}(\mathbf{r})$ the series (3.2), (3.3) and (3.4) of Lemma 3.1, respectively. Then we can prove the following theorem.

THEOREM 3.1. The total exterior field of the transmission problem (NHTP) has the integral representation

$$
\boldsymbol{\psi}_{0}(\mathbf{r})=\boldsymbol{\psi}(\mathbf{r})+\frac{1}{4 \pi} \boldsymbol{\sigma}_{1}(\mathbf{r})+\frac{\omega^{2}}{4 \pi} \boldsymbol{\sigma}_{2}(\mathbf{r})+\frac{\omega^{2}}{4 \pi} \boldsymbol{\sigma}_{3}(\mathbf{r}) .
$$

Pro of. As is well known [6], the scattered field $\mathbf{u}_{0}(\mathbf{r})$ has, in an infinite medium, the following integral representation:

$$
\mathbf{u}_{0}(\mathbf{r})=\frac{1}{4 \pi} \int_{S_{0}}\left[\mathbf{u}_{0}\left(\mathbf{r}^{\prime}\right) \cdot T_{0} \widetilde{G}_{0}\left(\mathbf{r}, \mathbf{r}^{\prime}\right)-\widetilde{G}_{0}\left(\mathbf{r}, \mathbf{r}^{\prime}\right) \cdot T_{0} \mathbf{u}_{0}\left(\mathbf{r}^{\prime}\right)\right] d s\left(\mathbf{r}^{\prime}\right) .
$$

The incident wave $\boldsymbol{\psi}$ is a solution of $\Delta^{*} \mathbf{u}+\omega^{2} \mathbf{u}=\mathbf{0}$, which has no singularities in $\mathbb{R}^{3}$. So, Betti's third formula implies that

$$
\int_{S_{0}}\left[\boldsymbol{\psi}\left(\mathbf{r}^{\prime}\right) \cdot T_{0} \widetilde{G}_{0}\left(\mathbf{r}, \mathbf{r}^{\prime}\right)-\widetilde{G}_{0}\left(\mathbf{r}, \mathbf{r}^{\prime}\right) \cdot T_{0} \boldsymbol{\psi}\left(\mathbf{r}^{\prime}\right)\right] d s\left(\mathbf{r}^{\prime}\right)=\mathbf{0} .
$$

From (2.3), (3.6) and (3.7) we conclude

$$
\boldsymbol{\psi}_{0}(\mathbf{r})=\boldsymbol{\psi}(\mathbf{r})+\frac{1}{4 \pi} \int_{S_{0}}\left[\boldsymbol{\psi}_{0}\left(\mathbf{r}^{\prime}\right) \cdot T_{0} \widetilde{G}_{0}\left(\mathbf{r}, \mathbf{r}^{\prime}\right)-\widetilde{G}_{0}\left(\mathbf{r}, \mathbf{r}^{\prime}\right) \cdot T_{0} \boldsymbol{\psi}_{0}\left(\mathbf{r}^{\prime}\right)\right] d s\left(\mathbf{r}^{\prime}\right) .
$$

Inserting the transmission conditions (2.11) on $S_{0}$ to (3.8), we obtain

$$
\begin{aligned}
\boldsymbol{\psi}_{0}(\mathbf{r})= & \boldsymbol{\psi}(\mathbf{r}) \\
& +\frac{1}{4 \pi} \int_{S_{0}}\left[\mathbf{u}_{1}\left(\mathbf{r}^{\prime}\right) \cdot T_{0} \widetilde{G}_{0}\left(\mathbf{r}, \mathbf{r}^{\prime}\right)-\widetilde{G}_{0}\left(\mathbf{r}, \mathbf{r}^{\prime}\right) \cdot T_{1} \mathbf{u}_{1}\left(\mathbf{r}^{\prime}\right)\right] d s\left(\mathbf{r}^{\prime}\right) .
\end{aligned}
$$


Applying successively Betti's third formula for $\mathbf{u}_{j}$ and $\widetilde{G}_{0}$ in $\Omega_{j}$, and using the transmission conditions (2.12), we get, for $j=1, \ldots, N$,

$$
\begin{aligned}
\boldsymbol{\psi}_{0}(\mathbf{r}) & =\boldsymbol{\psi}(\mathbf{r})+\frac{1}{4 \pi} \sum_{j=1}^{N} \int_{S_{j-1}} \mathbf{u}_{j}\left(\mathbf{r}^{\prime}\right) \cdot\left(T_{j-1}-T_{j}\right) \widetilde{G}_{0}\left(\mathbf{r}, \mathbf{r}^{\prime}\right) d s\left(\mathbf{r}^{\prime}\right) \\
+ & \frac{1}{4 \pi} \int_{S_{N}}\left[\mathbf{u}_{N}\left(\mathbf{r}^{\prime}\right) \cdot T_{N} \widetilde{G}_{0}\left(\mathbf{r}, \mathbf{r}^{\prime}\right)-\widetilde{G}_{0}\left(\mathbf{r}, \mathbf{r}^{\prime}\right) \cdot T_{N} \mathbf{u}_{N}\left(\mathbf{r}^{\prime}\right)\right] d s\left(\mathbf{r}^{\prime}\right) \\
& +\frac{1}{4 \pi} \sum_{j=1}^{N} \int_{\Omega_{j}}\left[\mathbf{u}_{j}\left(\mathbf{r}^{\prime}\right) \cdot \Delta_{j}^{*} \widetilde{G}_{0}\left(\mathbf{r}, \mathbf{r}^{\prime}\right)-\widetilde{G}_{0}\left(\mathbf{r}, \mathbf{r}^{\prime}\right) \cdot \Delta_{j}^{*} \mathbf{u}_{j}\left(\mathbf{r}^{\prime}\right)\right] d v\left(\mathbf{r}^{\prime}\right) .
\end{aligned}
$$

From the definition of $\Delta_{j}^{*}$, it is easy to show that

$$
\Delta_{j}^{*} \widetilde{G}_{0}\left(\mathbf{r}, \mathbf{r}^{\prime}\right)=-\omega^{2} \frac{\mu_{j}}{\mu_{0}} \widetilde{G}_{0}\left(\mathbf{r}, \mathbf{r}^{\prime}\right)+\left(\lambda_{j}-\frac{\lambda_{0}}{\mu_{0}} \mu_{j}\right) \operatorname{grad} \operatorname{div} \widetilde{G}_{0}\left(\mathbf{r}, \mathbf{r}^{\prime}\right) .
$$

Substituting (3.11) into (3.10), letting $N \rightarrow \infty$, and taking into account the convergence of the series in Lemma 3.1, we complete the proof.

As far as the scattering amplitudes are concerned, we have the following asymptotic relations, analogous to those of Barrat and Collins [4].

THEOREM 3.2. The scattered field of the transmission problem (NHTP) has the asymptotic behaviour

$$
\mathbf{u}_{0}(\mathbf{r})=\mathbf{g}^{\mathrm{p}}(\widehat{\mathbf{r}}, \widehat{\mathbf{k}}) h\left(k_{\mathrm{p}, 0} r\right)+\mathbf{g}^{\mathrm{s}}(\widehat{\mathbf{r}}, \widehat{\mathbf{k}}) h\left(k_{\mathrm{s}, 0} r\right)+O\left(1 / r^{2}\right), \quad r \rightarrow \infty,
$$

where the scattering amplitudes $\mathbf{g}^{\mathrm{p}}, \mathbf{g}^{\mathrm{s}}$ are given by

$$
\begin{aligned}
\mathbf{g}^{\mathrm{p}}(\widehat{\mathbf{r}}, \widehat{\mathbf{k}})= & \frac{1}{4 \pi\left(\lambda_{0}+2 \mu_{0}\right)}\left\{\sum _ { j = 1 } ^ { \infty } \left[2\left(\mu_{j-1}-\mu_{j}\right)\left(\widetilde{H}_{\mathrm{p}, j}: \widehat{\mathbf{r}} \otimes \widehat{\mathbf{r}}\right)\right.\right. \\
& +\left(\lambda_{j-1}-\lambda_{j}\right) \operatorname{SI}\left(\widetilde{H}_{\mathrm{p}, j}\right) \\
& \left.\left.+\omega^{2}\left(\widehat{\mathbf{r}} \cdot \mathbf{F}_{\mathrm{p}, j}\right)\left[1-\frac{\mu_{j}}{\mu_{0}}-k_{\mathrm{p}, 0}^{2}\left(\lambda_{j}-\frac{\lambda_{0}}{\mu_{0}} \mu_{j}\right)\right]\right]\right\} \widehat{\mathbf{r}}, \\
\mathbf{g}^{\mathrm{s}}(\widehat{\mathbf{r}}, \widehat{\mathbf{k}})= & \frac{1}{4 \pi \mu_{0}}\left\{\sum _ { j = 1 } ^ { \infty } \left[\left(\mu_{j-1}-\mu_{j}\right)\right.\right. \\
& \times\left[\widehat{\mathbf{r}} \cdot \widetilde{H}_{\mathrm{s}, j}+\widetilde{H}_{\mathrm{s}, j} \cdot \widehat{\mathbf{r}}-2\left(\widetilde{H}_{\mathrm{s}, j}: \widehat{\mathbf{r}} \otimes \widehat{\mathbf{r}}\right) \widehat{\mathbf{r}}\right] \\
& \left.\left.+\omega^{2}\left(1-\frac{\mu_{j}}{\mu_{0}}\right) \mathbf{F}_{\mathrm{s}, j} \cdot(\widetilde{I}-\widehat{\mathbf{r}} \otimes \widehat{\mathbf{r}})\right]\right\} .
\end{aligned}
$$

The quantities appearing above are given by $(\gamma=\mathrm{p}, \mathrm{s})$

$$
\widetilde{H}_{\gamma, j}=k_{\gamma, 0}^{2} \int_{S_{j-1}} \mathbf{u}_{j}\left(\mathbf{r}^{\prime}\right) \otimes \widehat{\mathbf{n}} \exp \left(-i k_{\gamma, 0} \widehat{\mathbf{r}} \cdot \mathbf{r}^{\prime}\right) d s\left(\mathbf{r}^{\prime}\right),
$$




$$
\mathbf{F}_{\gamma, j}=i k_{\gamma, 0} \int_{\Omega_{j}} \mathbf{u}_{j}\left(\mathbf{r}^{\prime}\right) \exp \left(-i k_{\gamma, 0} \widehat{\mathbf{r}} \cdot \mathbf{r}^{\prime}\right) d v\left(\mathbf{r}^{\prime}\right),
$$

$\operatorname{SI}\left(\widetilde{H}_{\gamma, j}\right)$ is the scalar invariant of the dyadic $\widetilde{H}_{\gamma, j}$, and the double inner product appearing in (3.13), (3.14) is defined as

$$
(\mathbf{a} \otimes \mathbf{b}):(\mathbf{c} \otimes \mathbf{d})=(\mathbf{a} \cdot \mathbf{d})(\mathbf{b} \cdot \mathbf{c}) .
$$

The function $\mathbf{g}^{\mathrm{p}}$ has the outgoing radial direction $\widehat{\mathbf{r}}$, and denotes the scattering amplitude of the longitudinal wave $\mathbf{u}^{\mathrm{p}}$. Also the function $\mathbf{g}^{\mathrm{s}}$ has a tangential direction, and denotes the scattering amplitude of the transverse wave $\mathbf{u}^{\mathrm{s}}$.

The proof of Theorem 3.2 follows by substituting (A63)-(A67) (Appendix) into (3.5).

4. Concluding remarks. For the proof of the existence of solutions of the transmission problem, we have used a generalized solutions approach. The standard approach, i.e. the implementation of potential theory, leads, in our case, to an infinite system of integral equations. Even in the case of a finite number of layers, the generalized solutions method does not present disadvantages as far as the length of the proof is concerned, in comparison to the standard method.

Consider the case

$$
\lambda_{j}=\lambda_{j+1}, \quad \mu_{j}=\mu_{j+1}, \quad j=q, q+1, \ldots, q \in \mathbb{N}_{0} .
$$

If $q=0$, no scattering occurs through $S_{j}$.

If $q=1$, the scatterer consists of only one layer. In this case, the problem has been quantitatively treated in [6]. Let $g_{r}, g_{\theta}, g_{\varphi}$ denote the normalized spherical scattering amplitudes which describe the effect of the scatterer in the directions $\widehat{\mathbf{r}}, \widehat{\boldsymbol{\theta}}, \widehat{\boldsymbol{\varphi}}$, respectively [6]. Their relation to the scattering amplitudes of Section 3 is given by $\mathbf{g}^{\mathrm{p}}=g_{r} \widehat{\mathbf{r}}$ and $\mathbf{g}^{\mathbf{s}}=g_{\theta} \widehat{\boldsymbol{\theta}}+g_{\varphi} \widehat{\boldsymbol{\varphi}}$.

The proofs of existence and uniqueness of solutions of the transmission problems in the above cases of $q=0$ and $q=1$ can be found in [12], and are performed by the potential theory method.

If $q=2$, the scatterer consists of only two layers. This case has been quantitatively studied in [11].

The quantitative treatment of the case where $3 \leq q<\infty$ is performed in [14] for low frequencies. A remark on the solvability of this transmission problem by the standard approach may be found in [10].

Acknowledgements. We are indebted to Professor S. A. Tersenov (Novosibirsk State University and Mathematical Institute of the Russian Academy of Sciences, Siberian Branch) for valuable discussions during the preparation of this work. 


\section{Appendix}

Lemma A.1. Let $U_{j}, K_{j}$ be the potential and kinetic energy, respectively, in $\Omega_{j}$. Then

$$
\sum_{j=1}^{\infty} \frac{\partial}{\partial t}\left(U_{j}+K_{j}\right)=\frac{\partial}{\partial t}\left[\sum_{j=1}^{\infty}\left(U_{j}+K_{j}\right)\right] .
$$

Proof. Let $\mathbf{u}(\mathbf{r})$ be a solution of (HTP). Since

$$
\mathbf{v}(\mathbf{r}, t)=\operatorname{Re}[\mathbf{u}(\mathbf{r}) \exp (-i \omega t)]
$$

if we set (as in [12])

$$
\begin{aligned}
& \mathbf{u}(\mathbf{r})=\mathbf{A}(\mathbf{r})+i \mathbf{B}(\mathbf{r}), \\
& \mathbf{A}(\mathbf{r})=\left(A_{1}(\mathbf{r}), A_{2}(\mathbf{r}), A_{3}(\mathbf{r})\right), \quad \mathbf{B}(\mathbf{r})=\left(B_{1}(\mathbf{r}), B_{2}(\mathbf{r}), B_{3}(\mathbf{r})\right),
\end{aligned}
$$

we get

$$
\mathbf{v}(\mathbf{r}, t)=\mathbf{A}(\mathbf{r}) \cos \omega t+\mathbf{B}(\mathbf{r}) \sin \omega t
$$

Let

$$
a(\mathbf{r})=\operatorname{div} \mathbf{A}(\mathbf{r}), \quad b(\mathbf{r})=\operatorname{div} \mathbf{B}(\mathbf{r})
$$

and

$$
\begin{aligned}
& A_{m q}(\mathbf{r})=\frac{1}{2}\left(\frac{\partial A_{m}(\mathbf{r})}{\partial x_{q}}+\frac{\partial A_{q}(\mathbf{r})}{\partial x_{m}}\right) \\
& B_{m q}(\mathbf{r})=\frac{1}{2}\left(\frac{\partial B_{m}(\mathbf{r})}{\partial x_{q}}+\frac{\partial B_{q}(\mathbf{r})}{\partial x_{m}}\right), \quad m, q=1,2,3 .
\end{aligned}
$$

Then

$$
\operatorname{div} \mathbf{v}(\mathbf{r}, t)=a(\mathbf{r}) \cos \omega t+b(\mathbf{r}) \sin \omega t
$$

and

$$
v_{m q}(\mathbf{r}, t)=A_{m q}(\mathbf{r}) \cos \omega t+B_{m q}(\mathbf{r}) \sin \omega t .
$$

We therefore have

$$
\begin{aligned}
U+K= & \int_{\Omega}\left\{\frac{1}{2} \lambda(a(\mathbf{r}) \cos \omega t+b(\mathbf{r}) \sin \omega t)^{2}\right. \\
& +\mu \sum_{m, q=1}^{3}\left(A_{m q}(\mathbf{r}) \cos \omega t+B_{m q}(\mathbf{r}) \sin \omega t\right)^{2} \\
& \left.+\omega \sum_{m=1}^{3}\left(B_{m}(\mathbf{r}) \cos \omega t-A_{m}(\mathbf{r}) \sin \omega t\right)^{2}\right\} d x
\end{aligned}
$$

and 


$$
\begin{aligned}
& \text { (A9) } \frac{\partial}{\partial t}(U+K) \\
& =\int_{\Omega}\{\lambda \omega(a(\mathbf{r}) \cos \omega t+b(\mathbf{r}) \sin \omega t)(b(\mathbf{r}) \cos \omega t-a(\mathbf{r}) \sin \omega t) \\
& \quad+2 \mu \omega \sum_{m, q=1}^{3}\left(A_{m q}(\mathbf{r}) \cos \omega t+B_{m q}(\mathbf{r}) \sin \omega t\right) \\
& \quad \times\left(B_{m q}(\mathbf{r}) \cos \omega t-A_{m q}(\mathbf{r}) \sin \omega t\right) \\
& \left.\quad-\omega^{2} \sum_{m=1}^{3}\left(B_{m}(\mathbf{r}) \cos \omega t-A_{m}(\mathbf{r}) \sin \omega t\right)\left(B_{m}(\mathbf{r}) \sin \omega t+A_{m}(\mathbf{r}) \cos \omega t\right)\right\} d x .
\end{aligned}
$$

The above will be considered in each $\Omega_{j}, j=1,2, \ldots$, and then a superscript " $(j)$ " will appear in the quantity involved.

Let $\lambda^{*}=\sup _{j} \lambda_{j}$ and $\mu^{*}=\sup _{j} \mu_{j}$. We assume that (in accordance to what is expected by physical considerations) $\lambda^{*}, \mu^{*}<\infty$. Let, moreover,

$$
\begin{cases}A_{* *}^{(j)}(\mathbf{r})=\max _{m, q} A_{m q}^{(j)}(\mathbf{r}), & \mathbf{r} \in \Omega_{j}, \\ B_{* *}^{(j)}(\mathbf{r})=\max _{m, q} B_{m q}^{(j)}(\mathbf{r}), & \mathbf{r} \in \Omega_{j},\end{cases}
$$

and

$$
\begin{cases}A_{*}^{(j)}(\mathbf{r})=\max _{m} A_{m}^{(j)}(\mathbf{r}), & \mathbf{r} \in \Omega_{j}, \\ B_{*}^{(j)}(\mathbf{r})=\max _{m} B_{m}^{(j)}(\mathbf{r}), & \mathbf{r} \in \Omega_{j},\end{cases}
$$

for $m, q=1,2,3$, and $j=1,2, \ldots$ Then, by (A8),

$$
\begin{aligned}
\left|U_{j}+K_{j}\right| \leq & \frac{1}{2} \lambda^{*} \int_{\Omega_{j}}\left(\left|a^{(j)}(\mathbf{r})\right|+\left|b^{(j)}(\mathbf{r})\right|\right)^{2} d x \\
& +3 \mu^{*} \int_{\Omega_{j}}\left(\left|A_{* *}^{(j)}(\mathbf{r})\right|+\left|B_{* *}^{(j)}(\mathbf{r})\right|\right)^{2} d x \\
& +3 \omega \int_{\Omega_{j}}\left(\left|A_{*}^{(j)}(\mathbf{r})\right|+\left|B_{*}^{(j)}(\mathbf{r})\right|\right)^{2} d x \\
\leq & \lambda^{*}\left\{\int_{\Omega_{j}}\left|a^{(j)}(\mathbf{r})\right|^{2} d x+\int_{\Omega_{j}}\left|b^{(j)}(\mathbf{r})\right|^{2} d x\right\} \\
& +6 \mu^{*}\left\{\int_{\Omega_{j}}\left|A_{* *}^{(j)}(\mathbf{r})\right|^{2} d x+\int_{\Omega_{j}}\left|B_{* *}^{(j)}(\mathbf{r})\right|^{2} d x\right\} \\
& +6 \omega\left\{\int_{\Omega_{j}}\left|A_{*}^{(j)}(\mathbf{r})\right|^{2} d x+\int_{\Omega_{j}}\left|B_{*}^{(j)}(\mathbf{r})\right|^{2} d x\right\},
\end{aligned}
$$


whence

$$
\begin{aligned}
\left|U_{j}+K_{j}\right| \leq & \lambda^{*}\left\{\left\|a^{(j)}\right\|_{L^{2}\left(\Omega_{j}\right)}^{2}+\left\|b^{(j)}\right\|_{L^{2}\left(\Omega_{j}\right)}^{2}\right\} \\
& +6 \mu^{*}\left\{\left\|A_{* *}^{(j)}\right\|_{L^{2}\left(\Omega_{j}\right)}^{2}+\left\|B_{* *}^{(j)}\right\|_{L^{2}\left(\Omega_{j}\right)}^{2}\right\} \\
& +6 \omega\left\{\left\|A_{*}^{(j)}\right\|_{L^{2}\left(\Omega_{j}\right)}^{2}+\left\|B_{*}^{(j)}\right\|_{L^{2}\left(\Omega_{j}\right)}^{2}\right\} .
\end{aligned}
$$

By (A4), (A5) and the definition of the $L^{2}$ and $H^{1}$ norms, (A13) gives

$$
\left|U_{j}+K_{j}\right| \leq c\left\{\left\|\mathbf{A}^{(j)}\right\|_{\left(H^{1}\left(\Omega_{j}\right)\right)^{3}}^{2}+\left\|\mathbf{B}^{(j)}\right\|_{\left(H^{1}\left(\Omega_{j}\right)\right)^{3}}^{2}\right\}
$$

where $c$ is a constant independent of $j$, depending only on $\lambda^{*}, \mu^{*}, \omega$.

Now, by the structure of our scatterer $\Omega$, we have

$$
\sum_{j=1}^{\infty}\left\|\boldsymbol{\varphi}^{(j)}\right\|_{\left(H^{1}\left(\Omega_{j}\right)\right)^{3}}^{2}=\left\|\boldsymbol{\varphi}^{(j)}\right\|_{\left(H^{1}(\Omega)\right)^{3}}^{2},
$$

whence

$$
\begin{aligned}
& \sum_{j=1}^{\infty}\left\|\mathbf{A}^{(j)}\right\|_{\left(H^{1}\left(\Omega_{j}\right)\right)^{3}}^{2}=\|\mathbf{A}\|_{\left(H^{1}(\Omega)\right)^{3}}^{2}=\|\operatorname{Re} \mathbf{u}\|_{\left(H^{1}(\Omega)\right)^{3}}^{2}, \\
& \sum_{j=1}^{\infty}\left\|\mathbf{B}^{(j)}\right\|_{\left(H^{1}\left(\Omega_{j}\right)\right)^{3}}^{2}=\|\mathbf{B}\|_{\left(H^{1}(\Omega)\right)^{3}}^{2}=\|\operatorname{Im} \mathbf{u}\|_{\left(H^{1}(\Omega)\right)^{3}}^{2},
\end{aligned}
$$

thus proving the uniform convergence of the series $\sum_{j=1}^{\infty}\left(U_{j}+K_{j}\right)$.

As far as $\sum_{j=1}^{\infty}(\partial / \partial t)\left(U_{j}+K_{j}\right)$ is concerned, the same conclusion holds. To prove it, we note that by (A9) we have

$$
\begin{aligned}
\left|\frac{\partial}{\partial t}\left(U_{j}+K_{j}\right)\right| \leq & 2 \omega \lambda^{*} \int_{\Omega_{j}}\left(\left|a^{(j)}(\mathbf{r})\right|^{2}+\left|b^{(j)}(\mathbf{r})\right|^{2}\right) d x \\
& +12 \omega \mu^{*} \int_{\Omega_{j}}\left(\left|A_{* *}^{(j)}(\mathbf{r})\right|^{2}+\left|B_{* *}^{(j)}(\mathbf{r})\right|^{2}\right) d x \\
& +6 \omega^{2} \int_{\Omega_{j}}\left(\left|A_{*}^{(j)}(\mathbf{r})\right|^{2}+\left|B_{*}^{(j)}(\mathbf{r})\right|^{2}\right) d x
\end{aligned}
$$

and then we argue as from (A12) onwards.

LEMMA A.2. The relation

$$
\frac{\partial}{\partial t}\left[\left(U_{0}+K_{0}\right)+\sum_{j=1}^{\infty}\left(U_{j}+K_{j}\right)\right]=\int_{r=R}\left(\frac{\partial \mathbf{v}}{\partial t} \cdot T_{0} \mathbf{v}\right) d s
$$

implies that $\mathbf{u}_{0}(\mathbf{r})=\mathbf{0}$ in $\Omega_{0}$.

Proof. Let

$$
\begin{aligned}
& \mathbf{u}_{0}^{\mathrm{p}}(\mathbf{r})=\mathbf{w}^{\mathrm{p}}(\mathbf{r})+i \mathbf{z}^{\mathrm{p}}(\mathbf{r}), \\
& \mathbf{u}_{0}^{\mathrm{s}}(\mathbf{r})=\mathbf{w}^{\mathrm{s}}(\mathbf{r})+i \mathbf{z}^{\mathrm{s}}(\mathbf{r})
\end{aligned}
$$


In what follows we make use of a number of asymptotic estimates for $\mathbf{w}^{\gamma}$, $\mathbf{z}^{\gamma}, T \mathbf{w}^{\gamma}+k_{\gamma} c_{\gamma}^{2} \mathbf{z}^{\gamma}, T \mathbf{z}^{\gamma}-k_{\gamma} c_{\gamma}^{2} \mathbf{w}^{\gamma}, \gamma=\mathrm{p}, \mathrm{s}$, and $\mathbf{w}^{\mathrm{p}} \cdot \mathbf{w}^{\mathrm{s}}, \mathbf{z}^{\mathrm{p}} \cdot \mathbf{z}^{\mathrm{s}}, \mathbf{w}^{\mathrm{p}} \cdot \mathbf{z}^{\mathrm{s}}, \mathbf{w}^{\mathrm{s}} \cdot \mathbf{z}^{\mathrm{p}}$ (where $c_{\gamma}$ is the phase velocity of the longitudinal $(\gamma=\mathrm{p})$ and transverse $(\gamma=\mathrm{s})$ wave) that can be found in [12], pp. 50-52.

Let $\mathbf{v}^{\mathrm{p}}, \mathbf{v}^{\mathrm{s}}$ be the potential and solenoidal components of $\mathbf{v}$, respectively; then

$$
\mathbf{v}=\mathbf{v}^{\mathrm{p}}+\mathbf{v}^{\mathrm{s}}
$$

and - in accordance to (A6) — we have

$$
\mathbf{v}^{\gamma}(\mathbf{r}, t)=\mathbf{w}^{\gamma}(\mathbf{r}) \cos \omega t+\mathbf{z}^{\gamma}(\mathbf{r}) \sin \omega t, \quad \gamma=\mathrm{p}, \mathrm{s} .
$$

Hence

$$
\text { (A22) } \begin{aligned}
\frac{\partial \mathbf{v}}{\partial t} \cdot T_{0} \mathbf{v} & =\sum_{\gamma=\mathrm{p}, \mathrm{s}} \sum_{\delta=\mathrm{p}, \mathrm{s}} \frac{\partial \mathbf{v}^{\gamma}}{\partial t} \cdot T_{0} \mathbf{v}^{\delta} \\
& =\sum_{\gamma=\mathrm{p}, \mathrm{s}} k_{\gamma} c_{\gamma}^{2} \omega\left(\mathbf{z}^{\gamma}(\mathbf{r}) \cos \omega t-\mathbf{w}^{\gamma}(\mathbf{r}) \sin \omega t\right)^{2}+o\left(1 / R^{2}\right) .
\end{aligned}
$$

Therefore (2.18) becomes

$$
\begin{aligned}
\frac{\partial}{\partial t}\left[\left(U_{0}+K_{0}\right)\right. & \left.+\sum_{j=1}^{\infty}\left(U_{j}+K_{j}\right)\right] \\
& =\sum_{\gamma=\mathrm{p}, \mathrm{s}} k_{\gamma} c_{\gamma}^{2} \omega \int_{r=R}\left(\mathbf{z}^{\gamma} \cos \omega t-\mathbf{w}^{\gamma} \sin \omega t\right)^{2} d s+o(1) .
\end{aligned}
$$

Using (A9) we have

$$
\begin{aligned}
\frac{\partial}{\partial t}\left[\left(U_{0}+K_{0}\right)\right. & \left.+\sum_{j=1}^{\infty}\left(U_{j}+K_{j}\right)\right] \\
= & \int_{\Omega_{0, R}} \lambda_{0} \omega\left(a^{(0)}(\mathbf{r}) \cos \omega t+b^{(0)}(\mathbf{r}) \sin \omega t\right) \\
& \times\left(b^{(0)}(\mathbf{r}) \cos \omega t-a^{(0)}(\mathbf{r}) \sin \omega t\right) d x \\
& +\int_{\Omega_{0, R}} 2 \mu_{0} \omega \sum_{m, q=1}^{3}\left(A_{m q}^{(0)}(\mathbf{r}) \cos \omega t+B_{m q}^{(0)}(\mathbf{r}) \sin \omega t\right) \\
& \times\left(B_{m q}^{(0)}(\mathbf{r}) \cos \omega t-A_{m q}^{(0)}(\mathbf{r}) \sin \omega t\right) d x \\
& -\int_{\Omega_{0, R}} \omega^{2} \sum_{m=1}^{3}\left(B_{m}^{(0)}(\mathbf{r}) \cos \omega t-A_{m}^{(0)}(\mathbf{r}) \sin \omega t\right)
\end{aligned}
$$




$$
\begin{aligned}
& \times\left(B_{m}^{(0)}(\mathbf{r}) \sin \omega t+A_{m}^{(0)}(\mathbf{r}) \cos \omega t\right) d x \\
& +\sum_{j=1}^{\infty}\left\{\int_{\Omega_{j}} \lambda_{j} \omega\left(a^{(j)}(\mathbf{r}) \cos \omega t+b^{(j)}(\mathbf{r}) \sin \omega t\right)\right. \\
& \times\left(b^{(j)}(\mathbf{r}) \cos \omega t-a^{(j)}(\mathbf{r}) \sin \omega t\right) d x \\
& +\int_{\Omega_{j}} 2 \mu_{j} \omega \sum_{m, q=1}^{3}\left(A_{m q}^{(j)}(\mathbf{r}) \cos \omega t+B_{m q}^{(j)}(\mathbf{r}) \sin \omega t\right) \\
& \times\left(B_{m q}^{(j)}(\mathbf{r}) \cos \omega t-A_{m q}^{(j)}(\mathbf{r}) \sin \omega t\right) d x \\
& -\int_{\Omega_{j}} \omega^{2} \sum_{m=1}^{3}\left(B_{m}^{(j)}(\mathbf{r}) \cos \omega t-A_{m}^{(j)}(\mathbf{r}) \sin \omega t\right) \\
& \left.\times\left(B_{m}^{(j)}(\mathbf{r}) \sin \omega t+A_{m}^{(j)}(\mathbf{r}) \cos \omega t\right) d x\right\} .
\end{aligned}
$$

We note that the RHS of (A24) changes sign. Indeed, we have

$$
\left.\frac{\partial}{\partial t}\left[\left(U_{0}+K_{0}\right)+\sum_{j=1}^{\infty}\left(U_{j}+K_{j}\right)\right]\right|_{t=0}=Q_{0}+\sum_{j=1}^{\infty} Q_{j}
$$

where

$$
\begin{array}{r}
Q_{0}=\int_{\Omega_{0, R}}\left\{\lambda_{0} \omega a^{(0)}(\mathbf{r}) b^{(0)}(\mathbf{r})+2 \mu_{0} \omega\right. \\
\sum_{m, q=1}^{3} A_{m q}^{(0)}(\mathbf{r}) B_{m q}^{(0)}(\mathbf{r}) \\
\left.-\omega^{2} \sum_{m=1}^{3} A_{m}^{(0)}(\mathbf{r}) B_{m}^{(0)}(\mathbf{r})\right\} d x
\end{array}
$$

and

$$
\begin{aligned}
Q_{j}=\int_{\Omega_{j}}\left\{\lambda_{j} \omega a^{(j)}(\mathbf{r}) b^{(j)}(\mathbf{r})+2 \mu_{j} \omega\right. & \sum_{m, q=1}^{3} A_{m q}^{(j)}(\mathbf{r}) B_{m q}^{(j)}(\mathbf{r}) \\
& \left.-\omega^{2} \sum_{m=1}^{3} A_{m}^{(j)}(\mathbf{r}) B_{m}^{(j)}(\mathbf{r})\right\} d x
\end{aligned}
$$

and we also have

$$
\left.\frac{\partial}{\partial t}\left[\left(U_{0}+K_{0}\right)+\sum_{j=1}^{\infty}\left(U_{j}+K_{j}\right)\right]\right|_{t=\pi /(2 \omega)}=-\left(Q_{0}+\sum_{j=1}^{\infty} Q_{j}\right) .
$$

Suppose now that $Q_{0}+\sum_{j=1}^{\infty} Q_{j} \neq 0$; without loss of generality we may assume it to be positive. Since the RHS of (A23) is non-negative for sufficiently 
large $R,(\mathrm{~A} 23)$ and (A28) imply that for $\gamma=\mathrm{p}, \mathrm{s}$,

$$
\lim _{R \rightarrow \infty} \int_{r=R}\left|\mathbf{w}^{\gamma}\right|^{2} d s=0
$$

uniformly, over all directions. But it is well known ([12], p. 53) that $\mathbf{w}^{\gamma}(\mathbf{r})$ is a solution of Helmholtz's equation

$$
\Delta \mathbf{w}^{\gamma}+k_{\gamma, 0}^{2} \mathbf{w}^{\gamma}=\mathbf{0}, \quad \gamma=\mathrm{p}, \mathrm{s} .
$$

By Rellich's lemma ([12], p. 53), we obtain

$$
\mathbf{w}^{\gamma}(\mathbf{r}) \equiv \mathbf{0}, \quad \gamma=\mathrm{p}, \mathrm{s} .
$$

But then, on account of (A23),

$$
\begin{aligned}
\frac{\partial}{\partial t}\left[\left(U_{0}+K_{0}\right)+\sum_{j=1}^{\infty}\left(U_{j}\right.\right. & \left.\left.+K_{j}\right)\right] \\
& =\sum_{\gamma=1}^{2} k_{\gamma, 0} c_{\gamma}^{2} \omega \cos ^{2} \omega t \int_{r=R}\left|\mathbf{z}^{\gamma}\right|^{2} d s+o(1)
\end{aligned}
$$

and the change of sign of the LHS implies

$$
\lim _{R \rightarrow \infty} \int_{r=R}\left|\mathbf{z}^{\gamma}\right|^{2} d s=0, \quad \gamma=\mathrm{p}, \mathrm{s},
$$

whence, as above,

$$
\mathbf{z}^{\gamma} \equiv \mathbf{0}, \quad \gamma=\mathrm{p}, \mathrm{s} .
$$

Hence

$$
\mathbf{u}_{0}^{\mathrm{p}}(\mathbf{r})=\mathbf{u}_{0}^{\mathrm{s}}(\mathbf{r})=\mathbf{0}
$$

and since $\mathbf{u}=\mathbf{u}^{\mathrm{p}}+\mathbf{u}^{\mathrm{s}}$ we have

$$
\mathbf{u}_{0}(\mathbf{r})=\mathbf{0},
$$

which is the desired result.

In the case $Q_{0}+\sum_{j=1}^{\infty} Q_{j}=0,(\mathrm{~A} 29)$ and (A33) follow immediately from (A23), and the proof follows as above.

Proof of Lemma 3.1. It is well known [12] that the solutions of the reduced Navier equation in a bounded domain are bounded. Hence there exists $b>0$ such that

$$
\begin{aligned}
\left\|\mathbf{u}_{j}\left(\mathbf{r}^{\prime}\right)\right\| \leq b & \text { for } \mathbf{r}^{\prime} \in \Omega_{j}, j=1,2, \ldots, \\
\left\|\widetilde{G}_{0}\left(\mathbf{r}, \mathbf{r}^{\prime}\right)\right\|_{\mathrm{D}} \leq b & \text { for } \mathbf{r}^{\prime} \in \Omega_{j}, j=1,2, \ldots, \mathbf{r} \in \Omega_{0},
\end{aligned}
$$

where the norm, $\|\cdot\|_{\mathrm{D}}$, of a dyadic is defined as $\|\mathbf{v} \otimes \mathbf{w}\|_{\mathrm{D}}^{2}=\sum_{i, j=1}^{3}\left(v_{i} w_{j}\right)^{2}$.

In order to apply the surface stress operator

$$
T_{j}=2 \mu_{j} \widehat{\mathbf{n}} \cdot \operatorname{grad}+\lambda_{j} \widehat{\mathbf{n}} \operatorname{div}+\mu_{j} \widehat{\mathbf{n}} \times \operatorname{curl}
$$


to $\widetilde{G}_{0}\left(\mathbf{r}, \mathbf{r}^{\prime}\right)$, it is necessary to evaluate the gradient of $\widetilde{G}_{0}\left(\mathbf{r}, \mathbf{r}^{\prime}\right)$ with respect to the variable $\mathbf{r}^{\prime}$. We have

$$
\begin{aligned}
&(\mathrm{A} 40) \quad \operatorname{grad} \widetilde{G}_{0}\left(\mathbf{r}, \mathbf{r}^{\prime}\right) \\
&=-\frac{\exp \left(i k_{\mathrm{p}, 0} R\right)}{\left(\lambda_{0}+2 \mu_{0}\right) R}\left[M_{\mathrm{p}, 1}(R) \widehat{\mathbf{R}} \otimes \widehat{\mathbf{R}} \otimes \widehat{\mathbf{R}}+M_{\mathrm{p}, 2}(R) \widehat{\mathbf{R}} \otimes \widetilde{I}+M_{\mathrm{p}, 3}(R) \widetilde{I} \otimes \widehat{\mathbf{R}}\right] \\
&+\frac{\exp \left(i k_{\mathrm{s}, 0} R\right)}{\mu_{0} R}\left[M_{\mathrm{s}, 1}(R) \widehat{\mathbf{R}} \otimes \widehat{\mathbf{R}} \otimes \widehat{\mathbf{R}}\right. \\
&\left.+\left(M_{\mathrm{s}, 2}(R)-M_{\mathrm{s}, 4}(R)\right) \widehat{\mathbf{R}} \otimes \widetilde{I}+M_{\mathrm{s}, 3}(R) \widetilde{I} \otimes \widehat{\mathbf{R}}\right],
\end{aligned}
$$

where

$$
\begin{aligned}
& M_{\gamma, 1}(R)=i k_{\gamma, 0}-\frac{6}{R}-\frac{15 i}{k_{\gamma, 0} R^{2}}+\frac{15}{k_{\gamma, 0}^{2} R^{3}}, \\
& M_{\gamma, 2}(R)=\frac{2}{R}+\frac{6 i}{k_{\gamma, 0} R^{2}}-\frac{6}{k_{\gamma, 0}^{2} R^{3}}, \\
& M_{\gamma, 3}(R)=\frac{1}{R}+\frac{3 i}{k_{\gamma, 0} R^{2}}-\frac{3}{k_{\gamma, 0}^{2} R^{3}},
\end{aligned}
$$

with $\gamma=\mathrm{p}$, s for the longitudinal and transverse wave respectively, and

$$
M_{\mathrm{s}, 4}(R)=i k_{\mathrm{s}, 0}-\frac{1}{R} .
$$

Since $R \geq \theta^{-1}$, there exist $B_{\gamma, q}, q=1,2,3$, and $B_{\mathrm{s}, 4}$ such that

$$
\left|M_{\gamma, q}(R)\right| \leq B_{\gamma, q} \quad \text { and } \quad\left|M_{\mathrm{s}, 4}(R)\right| \leq B_{\mathrm{s}, 4} .
$$

In the triadic (A40) we take the scalar and the vector invariants between the first two vectors. So, we have

(A46) $\operatorname{div} \widetilde{G}_{0}\left(\mathbf{r}, \mathbf{r}^{\prime}\right)=-\frac{\exp \left(i k_{\mathrm{p}, 0} R\right)}{\left(\lambda_{0}+2 \mu_{0}\right) R}\left[M_{\mathrm{p}, 1}(R)+M_{\mathrm{p}, 2}(R)+3 M_{\mathrm{p}, 3}(R)\right] \widehat{\mathbf{R}}$,

(A47) $\operatorname{curl} \widetilde{G}_{0}\left(\mathbf{r}, \mathbf{r}^{\prime}\right)=-\frac{\exp \left(i k_{\mathrm{p}, 0} R\right)}{\left(\lambda_{0}+2 \mu_{0}\right) R} M_{\mathrm{p}, 2}(R) \widehat{\mathbf{R}} \times \widetilde{I}$

$$
+\frac{\exp \left(i k_{\mathrm{s}, 0} R\right)}{\mu_{0} R}\left(M_{\mathrm{s}, 2}(R)-M_{\mathrm{s}, 4}(R)\right) \widehat{\mathbf{R}} \otimes \widetilde{I} .
$$

Application of the surface stress operator $T_{j-1}-T_{j}$ to $\widetilde{G}_{0}\left(\mathbf{r}, \mathbf{r}^{\prime}\right)$ gives

$$
\begin{aligned}
\left(T_{j-1}-T_{j}\right) \widetilde{G}_{0}\left(\mathbf{r}, \mathbf{r}^{\prime}\right)= & 2\left(\mu_{j-1}-\mu_{j}\right) \widehat{\mathbf{n}} \operatorname{div} \widetilde{G}_{0}\left(\mathbf{r}, \mathbf{r}^{\prime}\right) \\
& +\left(\lambda_{j-1}-\lambda_{j}\right) \widehat{\mathbf{n}} \operatorname{div} \widetilde{G}_{0}\left(\mathbf{r}, \mathbf{r}^{\prime}\right) \\
& +\left(\mu_{j-1}-\mu_{j}\right) \widehat{\mathbf{n}} \times \operatorname{curl} \widetilde{G}_{0}\left(\mathbf{r}, \mathbf{r}^{\prime}\right) .
\end{aligned}
$$

From (A40), (A45), (A46) and (A47) we have

$$
\left\|\widehat{\mathbf{n}} \cdot \operatorname{grad} \widetilde{G}_{0}\left(\mathbf{r}, \mathbf{r}^{\prime}\right)\right\|_{\mathrm{D}} \leq C_{1},
$$




$$
\begin{aligned}
\left\|\widehat{\mathbf{n}} \operatorname{div} \widetilde{G}_{0}\left(\mathbf{r}, \mathbf{r}^{\prime}\right)\right\|_{\mathrm{D}} & \leq C_{2}, \\
\left\|\widehat{\mathbf{n}} \times \operatorname{curl} \widetilde{G}_{0}\left(\mathbf{r}, \mathbf{r}^{\prime}\right)\right\|_{\mathrm{D}} & \leq C_{3},
\end{aligned}
$$

where

$$
\begin{aligned}
C_{1} & =\frac{\theta}{\lambda_{0}+2 \mu_{0}} \sum_{q=1}^{3} B_{\mathrm{p}, q}+\frac{\theta}{\mu_{0}} B_{\mathrm{s}, q}, \\
C_{2} & =\frac{\theta}{\lambda_{0}+2 \mu_{0}} \sum_{q=1}^{3} B_{\mathrm{p}, q}, \\
C_{3} & =\frac{\theta}{\lambda_{0}+2 \mu_{0}} 4 B_{\mathrm{p}, 2}+\frac{\theta}{\mu_{0}}\left(B_{\mathrm{s}, 2}+B_{\mathrm{s}, 4}\right) .
\end{aligned}
$$

Using (A48)-(A51) we obtain the following estimate:

$$
\left\|\left(T_{j-1}-T_{j}\right) \widetilde{G}_{0}\left(\mathbf{r}, \mathbf{r}^{\prime}\right)\right\|_{\mathrm{D}} \leq 4 \mu^{*} C_{1}+2 \lambda^{*} C_{2}+2 \mu^{*} C_{3} \equiv B,
$$

where $\mu^{*}=\sup _{j} \mu_{j}$ and $\lambda^{*}=\sup _{j} \lambda_{j}, j=1,2, \ldots$ So, we have

$$
\left\|\int_{S_{j-1}} \mathbf{u}_{j}\left(\mathbf{r}^{\prime}\right) \cdot\left(T_{j-1}-T_{j}\right) \widetilde{G}_{0}\left(\mathbf{r}, \mathbf{r}^{\prime}\right) d s\left(\mathbf{r}^{\prime}\right)\right\| \leq b B\left|S_{j-1}\right| .
$$

From (A56), taking into account that $\sum_{j=0}^{\infty}\left|S_{j}\right|<\infty$, and using the Weierstrass M-test, we establish the uniform convergence of the series (3.2).

Also, from (A37), (A38) we have

$$
\left\|\left(1-\frac{\mu_{j}}{\mu_{0}}\right) \int_{\Omega_{j}} \mathbf{u}_{j}\left(\mathbf{r}^{\prime}\right) \cdot \widetilde{G}_{0}\left(\mathbf{r}, \mathbf{r}^{\prime}\right) d v\left(\mathbf{r}^{\prime}\right)\right\| \leq\left(1+\frac{\mu^{*}}{\mu_{0}}\right) b^{2}\left|\Omega_{j}\right| .
$$

Since, by the structure of the scatterer, we have $\sum_{j=1}^{\infty}\left|\Omega_{j}\right|=|\Omega|$, the series (3.3) converges uniformly.

Finally, since

(A58) $\operatorname{grad} \operatorname{div} \widetilde{G}_{0}\left(\mathbf{r}, \mathbf{r}^{\prime}\right)$

$$
=\frac{\exp \left(i k_{\mathrm{p}, 0} R\right)}{\left(\lambda_{0}+2 \mu_{0}\right) R}\left[\left(i k_{\mathrm{p}, 0}-\frac{1}{R}\right) \sum_{q=1}^{3} M_{\mathrm{p}, q}(R)+\frac{d}{d R} M_{\mathrm{p}, q}(R)\right] \widehat{\mathbf{R}} \otimes \widehat{\mathbf{R}},
$$

we see that there exists $B^{\prime}>0$ such that

$$
\left\|\operatorname{grad} \operatorname{div} \widetilde{G}_{0}\left(\mathbf{r}, \mathbf{r}^{\prime}\right)\right\|_{\mathrm{D}} \leq B^{\prime}
$$

So, we have

$$
\begin{aligned}
\text { (A60) }\left\|\left(\lambda_{j}-\frac{\lambda_{0}}{\mu_{0}} \mu_{j}\right) \int_{\Omega_{j}} \mathbf{u}_{j}\left(\mathbf{r}^{\prime}\right) \cdot\left[\operatorname{grad} \operatorname{div} \widetilde{G}_{0}\left(\mathbf{r}, \mathbf{r}^{\prime}\right)\right] d v\left(\mathbf{r}^{\prime}\right)\right\| \\
\leq\left(\lambda^{*}+\left(\lambda_{0} / \mu_{0}\right) \mu^{*}\right) b B^{\prime}\left|\Omega_{j}\right|,
\end{aligned}
$$

which ensures the uniform convergence of the series (3.4). 
Some useful asymptotic formulae. In the radiation region, using the asymptotic relations

$$
\begin{aligned}
\mathbf{R} / R & =\widehat{\mathbf{r}}+O(1 / r), & & r \rightarrow \infty, \\
R & =r-\widehat{\mathbf{r}} \cdot \mathbf{r}^{\prime}+O(1 / r), & & r \rightarrow \infty,
\end{aligned}
$$

we obtain the following asymptotic formulae for $r \rightarrow \infty$ :

$$
\begin{aligned}
\widetilde{G}_{0}\left(\mathbf{r}, \mathbf{r}^{\prime}\right)= & \frac{i k_{\mathrm{p}, 0}}{\lambda_{0}+2 \mu_{0}} \exp \left(-i k_{\mathrm{p}, 0} \widehat{\mathbf{r}} \cdot \mathbf{r}^{\prime}\right) h\left(k_{\mathrm{p}, 0} r\right) \widehat{\mathbf{r}} \otimes \widehat{\mathbf{r}} \\
& +\frac{i k_{\mathrm{s}, 0}}{\mu_{0}} \exp \left(-i k_{\mathrm{s}, 0} \widehat{\mathbf{r}} \cdot \mathbf{r}^{\prime}\right) h\left(k_{\mathrm{s}, 0} r\right)(\widetilde{I}-\widehat{\mathbf{r}} \otimes \widehat{\mathbf{r}}) \\
& +O\left(1 / r^{2}\right), \\
\operatorname{grad} \widetilde{G}_{0}\left(\mathbf{r}, \mathbf{r}^{\prime}\right)= & \frac{k_{\mathrm{p}, 0}^{2}}{\lambda_{0}+2 \mu_{0}} \exp \left(-i k_{\mathrm{p}, 0} \widehat{\mathbf{r}} \cdot \mathbf{r}^{\prime}\right) h\left(k_{\mathrm{p}, 0} r\right) \widehat{\mathbf{r}} \otimes \widehat{\mathbf{r}} \otimes \widehat{\mathbf{r}} \\
& +\frac{k_{\mathrm{s}, 0}^{2}}{\mu_{0}} \exp \left(-i k_{\mathrm{s}, 0} \widehat{\mathbf{r}} \cdot \mathbf{r}^{\prime}\right) h\left(k_{\mathrm{s}, 0} r\right) \widehat{\mathbf{r}} \otimes(\widetilde{I}-\widehat{\mathbf{r}} \otimes \widehat{\mathbf{r}}) \\
& +O\left(1 / r^{2}\right), \\
\operatorname{div} \widetilde{G}_{0}\left(\mathbf{r}, \mathbf{r}^{\prime}\right)= & \frac{k_{\mathrm{p}, 0}^{2}}{\lambda_{0}+2 \mu_{0}} \exp \left(-i k_{\mathrm{p}, 0} \widehat{\mathbf{r}} \cdot \mathbf{r}^{\prime}\right) h\left(k_{\mathrm{p}, 0} r\right) \widehat{\mathbf{r}}+O\left(1 / r^{2}\right), \\
\operatorname{curl} \widetilde{G}_{0}\left(\mathbf{r}, \mathbf{r}^{\prime}\right)= & \frac{k_{\mathrm{s}, 0}^{2}}{\mu_{0}} \exp \left(-i k_{\mathrm{s}, 0} \widehat{\mathbf{r}} \cdot \mathbf{r}^{\prime}\right) h\left(k_{\mathrm{s}, 0} r\right) \widehat{\mathbf{r}} \times \widetilde{I}+O\left(1 / r^{2}\right),
\end{aligned}
$$

$\operatorname{grad} \operatorname{div} \widetilde{G}_{0}\left(\mathbf{r}, \mathbf{r}^{\prime}\right)=-\frac{i k_{\mathrm{p}, 0}^{3}}{\lambda_{0}+2 \mu_{0}} \exp \left(-i k_{\mathrm{p}, 0} \widehat{\mathbf{r}} \cdot \mathbf{r}^{\prime}\right) h\left(k_{\mathrm{p}, 0} r\right) \widehat{\mathbf{r}} \otimes \widehat{\mathbf{r}}$

$$
+O\left(1 / r^{2}\right)
$$

where $h(x)=e^{i x} /(i x)$ is the zeroth order spherical Hankel function of the first kind.

\section{References}

[1] C. Athanasiadis and I. G. Stratis, Low-frequency acoustic scattering by an infinitely stratified scatterer, Rend. Mat. Appl. 15 (1995), 133-152.

[2] -, -, Parabolic and hyperbolic diffraction problems, Math. Japon. 43 (1996), 37-45.

[3] —, - On some elliptic transmission problems, Ann. Polon. Math. 63 (1996), 137154.

[4] P. J. Barrat and W. D. Collins, The scattering cross-section of an obstacle in an elastic solid for plane harmonic waves, Proc. Cambridge Philos. Soc. 61 (1965), 969-981.

[5] G. Caviglia and A. Morro, Inhomogeneous Waves in Solids and Fluids, World Sci., London, 1992.

[6] G. Dassios and K. Kiriaki, The low-frequency theory of elastic wave scattering, Quart. Appl. Math. 42 (1984), 225-248. 
[7] R. Dautray and J. L. Lions, Mathematical Analysis and Numerical Methods for Science and Technology, Vol. 1, Physical Origins and Classical Methods, Springer, Berlin, 1990.

[8] G. Fichera, Existence theorems in elasticity, in: Handbuch der Physik, Via/2, Springer, Berlin, 1972, 347-389.

[9] D. S. Jones, Low-frequency scattering in elasticity, Quart. J. Mech. Appl. Math. 34 (1981), 431-451.

[10] —, A uniqueness theorem in elastodynamics, ibid. 37 (1984), 121-142.

[11] K. Kiriaki and D. Polyzos, The low-frequency scattering theory for a penetrable scatterer with an impenetrable core in an elastic medium, Internat. J. Engrg. Sci. 26 (1988), 1143-1160.

[12] V. D. Kupradze, Potential Methods in the Theory of Elasticity, Israel Program for Scientific Translations, Jerusalem, 1965.

[13] V. D. Kupradze (ed.), Three-Dimensional Problems of the Mathematical Theory of Elasticity and Thermoelasticity, North-Holland, Amsterdam, 1979.

[14] D. Polyzos, Low-frequency elastic scattering theory for a multi-layered scatterer with dyadic incidence, submitted.

[15] P. C. Sabatier, On the scattering by discontinuous media, in: Inverse Problems in Partial Differential Equations, D. Colton, R. Ewing, W. Rundell (eds.), SIAM, Philadelphia, 1990, 85-100.

[16] L. T. Wheeler and E. Sternberg, Some theorems in classical elastodynamics, Arch. Rational Mech. Anal. 31 (1968), 51-90.

Department of Mathematics

University of Athens

Panepistemiopolis

GR-157 84 Athens, Greece

E-mail: istratis@eudoxos.uoa.gr 